Supplement of Biogeosciences, 13, 3777-3791, 2016

http://www.biogeosciences.net/13/3777/2016/

doi:10.5194/bg-13-3777-2016-supplement

(C) Author(s) 2016. CC Attribution 3.0 License.

(c) (i)

Supplement of

\title{
Summer fluxes of methane and carbon dioxide from a pond and floating mat in a continental Canadian peatland
}

Magdalena Burger et al.

Correspondence to: Christian Blodau (christian.blodau@uni-muenster.de)

The copyright of individual parts of the supplement might differ from the CC-BY 3.0 licence. 
11 Chamber flux measurements

2

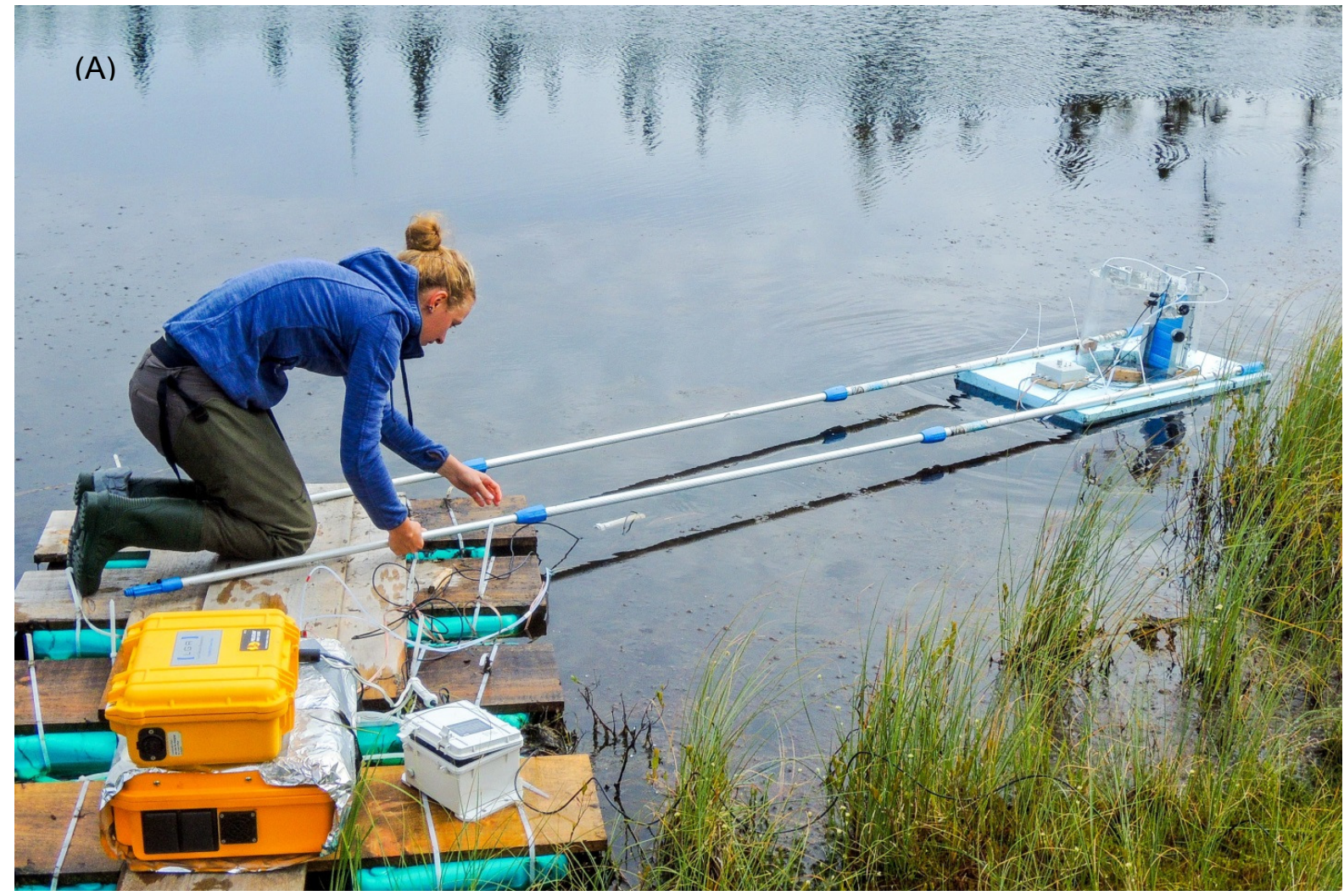

12

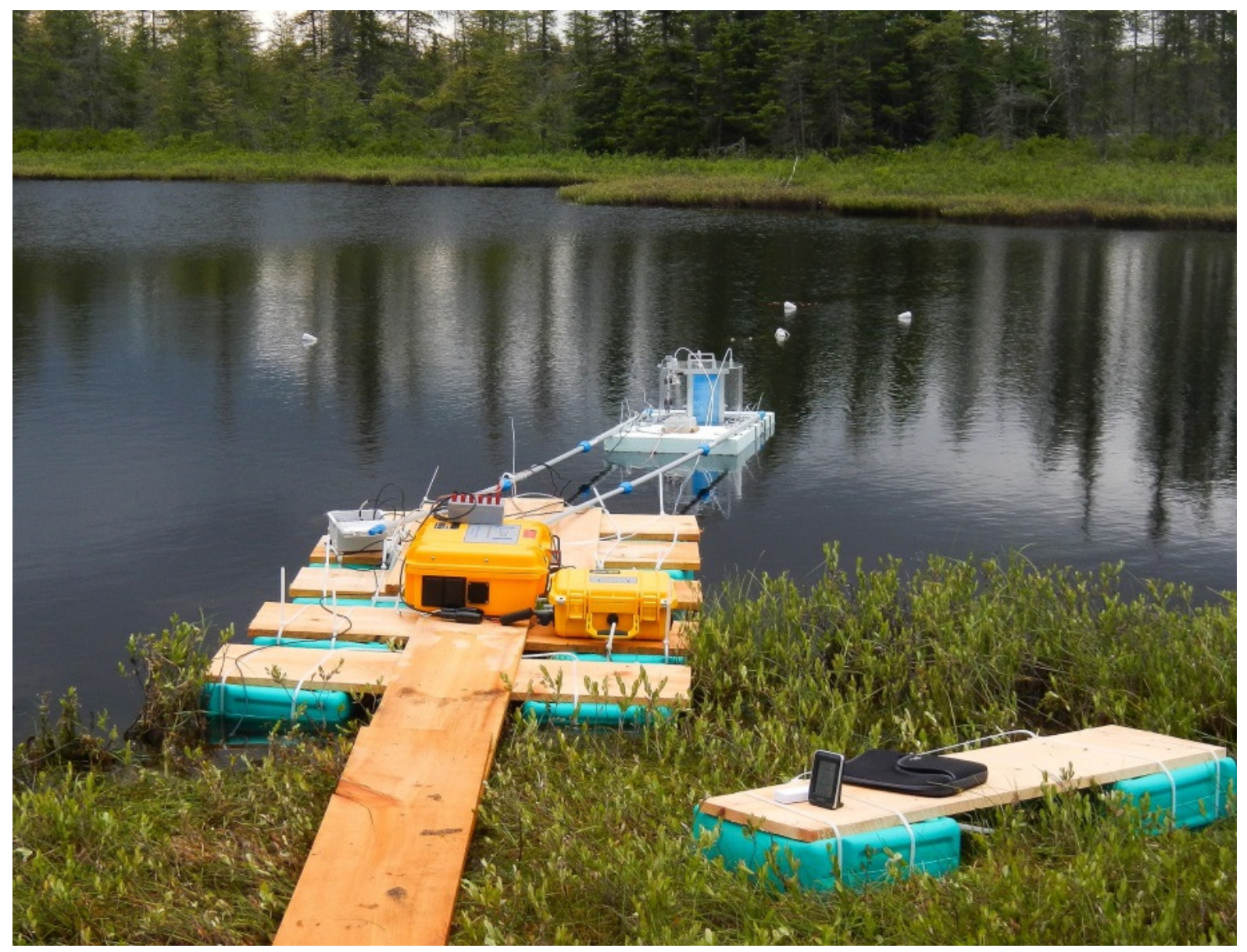



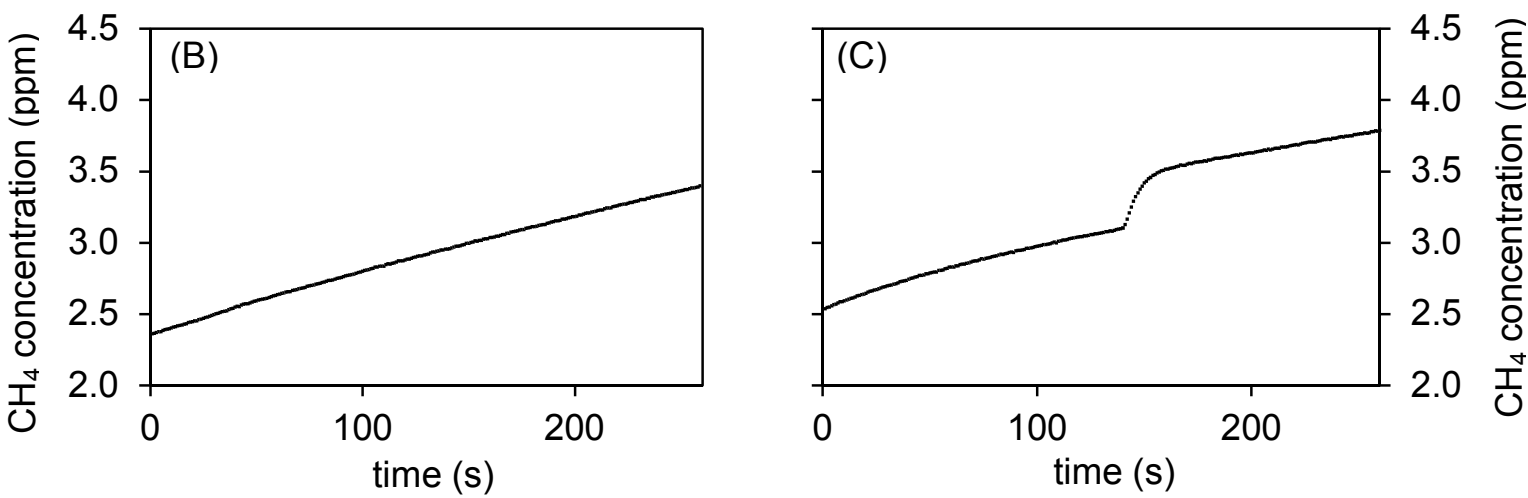

3 Figure S1. Set-up of flux measurements on the pond including the floating boardwalk and 4 chamber (A). Example of a concentration time series with a constant slope representing 5 diffusive $\mathrm{CH}_{4}$ flux or continuous flux of micro-bubbles (panel B) and example of a 6 concentration time series including a $\mathrm{CH}_{4}$ bubble event visible by a sudden increase of slope 7 over a short duration (panel C).

8

\section{2. $\mathrm{CO}_{2}$ flux calculation with the gradient method}

$\mathrm{CO}_{2}$ fluxes across the air-water interface were estimated according to the boundary layer equation

11 approach, equation

12

$$
\begin{array}{cl}
\mathrm{F}=\mathrm{k}_{\mathrm{CO}_{2}} & \cdot\left(\mathrm{c}_{\text {water }}-\mathrm{c}_{\mathrm{atm}}\right) \\
\mathrm{F} & \mathrm{CO}_{2} \text { flux }\left(\mathrm{mmol} \mathrm{m} \mathrm{m}^{-2} \mathrm{~h}^{-1}\right) \\
\mathrm{k}_{\mathrm{CO}_{2}} & \text { gas transfer velocity }\left(\mathrm{m} \mathrm{h}^{-1}\right) \\
\mathrm{c}_{\mathrm{water}} & \mathrm{CO}_{2} \text { concentration in the surface } \\
& \text { water }\left(\mathrm{mmol} \mathrm{m}{ }^{-3}\right) \\
\mathrm{c}_{\mathrm{atm}} & \text { theoretical } \mathrm{CO}_{2} \text { concentration of } \\
& \text { the surface water in equilibrium } \\
& \text { with the atmospheric } \mathrm{CO}_{2} \\
& \text { concentration }\left(\mathrm{mmol} \mathrm{m}^{-3}\right)
\end{array}
$$

$13 \mathrm{c}_{\text {water }}$ and $\mathrm{c}_{\mathrm{atm}}$ were calculated with Henry's law from the $\mathrm{CO}_{2}$ mixing ratios measured in the surface water and the atmosphere using Henry's law constants (Sander, 1999) corrected for surface water temperature. The gas transfer velocity $\mathrm{k}_{\mathrm{CO}_{2}}$ was calculated from the gas transfer velocity normalized to a Schmidt number of $600\left(\mathrm{k}_{600}\right)$ according to equation (2) (Crusius and Wanninkhof, 2003).

$17 \quad \mathrm{k}_{\mathrm{CO}_{2}}=\mathrm{k}_{600} \cdot\left(\frac{\mathrm{Sc}_{\mathrm{CO}_{2}}}{600}\right)^{-\mathrm{b}}$

$$
\begin{aligned}
& \mathrm{k}_{\mathrm{CO}_{2}} \text { gas transfer velocity }\left(\mathrm{m} \mathrm{h}^{-1}\right) \\
& \mathrm{k}_{600} \text { gas transfer velocity normalized } \\
& \text { to a Schmidt number of } 600 \\
& \left(\mathrm{~m} \mathrm{~h}^{-1}\right) \\
& \mathrm{Sc}_{\mathrm{CO}_{2}} \text { Schmidt number for } \mathrm{CO}_{2} \\
& \text { corrected for temperature (-) } \\
& 600 \text { Schmidt number of } \mathrm{CO}_{2} \text { at } 20^{\circ} \mathrm{C}
\end{aligned}
$$


B $\frac{1}{2}$ for wind speed $>3 \mathrm{~m} \mathrm{~s}^{-1}$
$\frac{2}{3}$ for wind speed $\leq 3 \mathrm{~m} \mathrm{~s}^{-1}$

$1 \mathrm{k}_{600}$ depends on the wind speed at a height of $10 \mathrm{~m}$ according to the empirical relationship defined

2 by Cole and Caraco (1998) (equation 3). $\mathrm{Sc}_{\mathrm{CO}_{2}}$ was calculated as a function of water temperature

3 (equation 5; Wanninkhof, 1992).

4

$5 \quad \mathrm{k}_{600}=0.0207+0.00215 \cdot \mathrm{U}_{10}{ }^{1.7}$

$\mathrm{k}_{600}$ gas transfer velocity normalized to a Schmidt number of $600\left(\mathrm{~m} \mathrm{~h}^{-1}\right)$

$\mathrm{U}_{10} \quad$ wind speed at $10 \mathrm{~m}$ height $\left(\mathrm{m} \mathrm{s}^{-1}\right)$

$6 \quad \mathrm{Sc}_{\mathrm{CO}_{2}}=1911.1-118.11 \cdot \mathrm{T}+3.4527 \cdot$

$\mathrm{Sc}_{\mathrm{CO}_{2}}$ Schmidt number for $\mathrm{CO}_{2}$ corrected for temperature (-)

$\mathrm{T} \quad$ water temperature $\left({ }^{\circ} \mathrm{C}\right)$

7 Wind speed at a height of $10 \mathrm{~m}$ was estimated from the wind speed measurements at the weather

8 station at a height of $2.5 \mathrm{~m}$ according to equation (5) (Singh et al., 2007).

$9 \quad \mathrm{U}_{2}=\mathrm{U}_{1} \cdot\left(\frac{\mathrm{h}_{2}}{\mathrm{~h}_{1}}\right)^{\frac{1}{6}}$

$\mathrm{U}_{2} \quad$ estimated wind speed $\left(\mathrm{m} \mathrm{s}^{-1}\right)$ at $\mathrm{h}_{2}$

$\mathrm{U}_{1} \quad$ measured wind speed $\left(\mathrm{m} \mathrm{s}^{-1}\right)$ at $\mathrm{h}_{1}$

$\mathrm{h}_{2} \quad$ height $(\mathrm{m})$ of the estimated wind speed $\mathrm{U}_{2} ; 10 \mathrm{~m}$

$\mathrm{h}_{1} \quad$ height $(\mathrm{m})$ of the measured wind speed $\mathrm{U}_{1} ; 2.5 \mathrm{~m}$ 

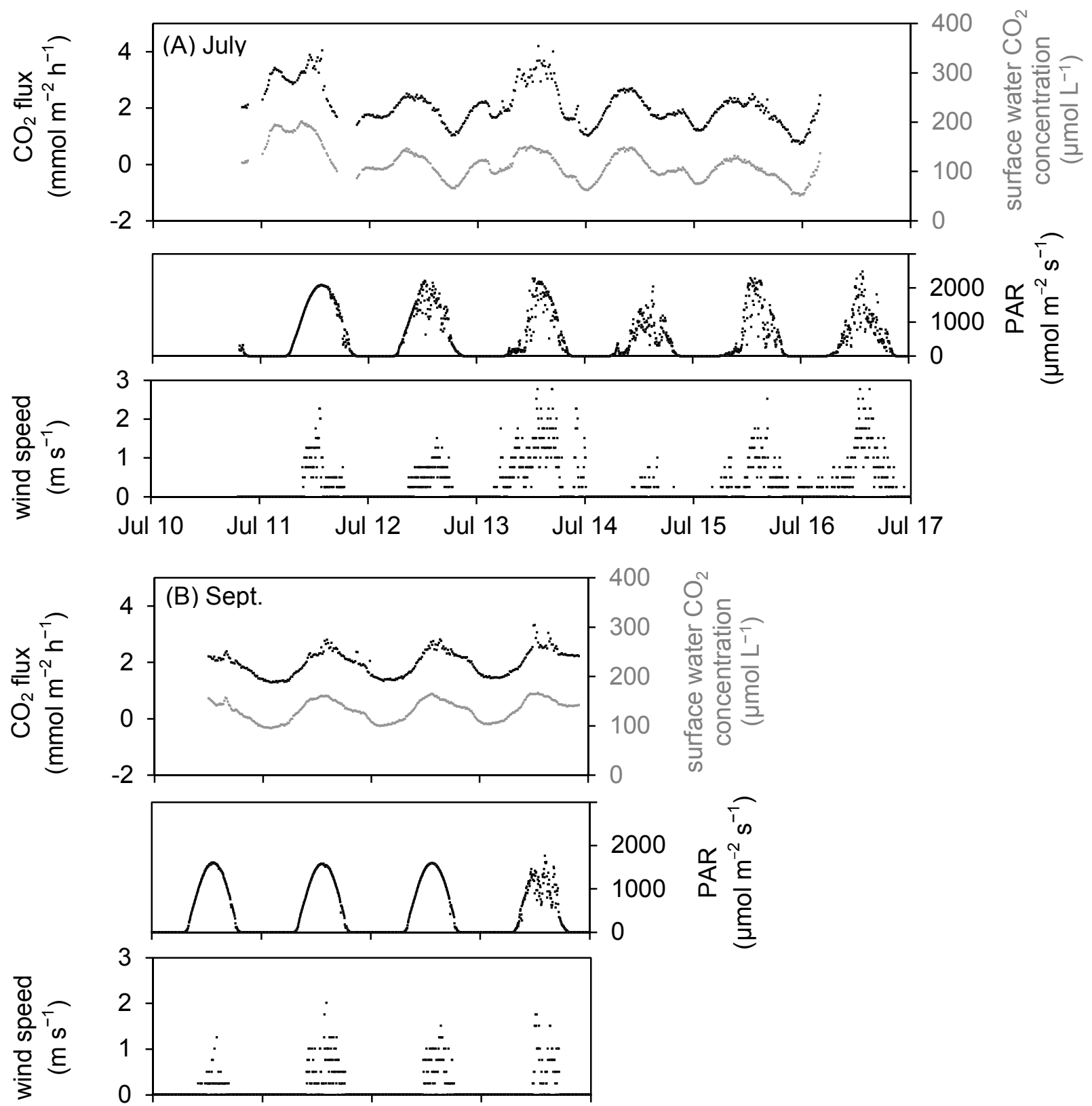

Sep $25 \quad$ Sep $26 \quad$ Sep $27 \quad$ Sep $28 \quad$ Sep 29

Figure S2: $\mathrm{CO}_{2}$ concentrations in the surface water of the pond (gray symbols), $\mathrm{CO}_{2}$ fluxes calculated with the gradient method (black symbols), photosynthetically active radiation and wind speed (both 5 min averages) during two periods in July (panel A) and September (panel B) 2014. 


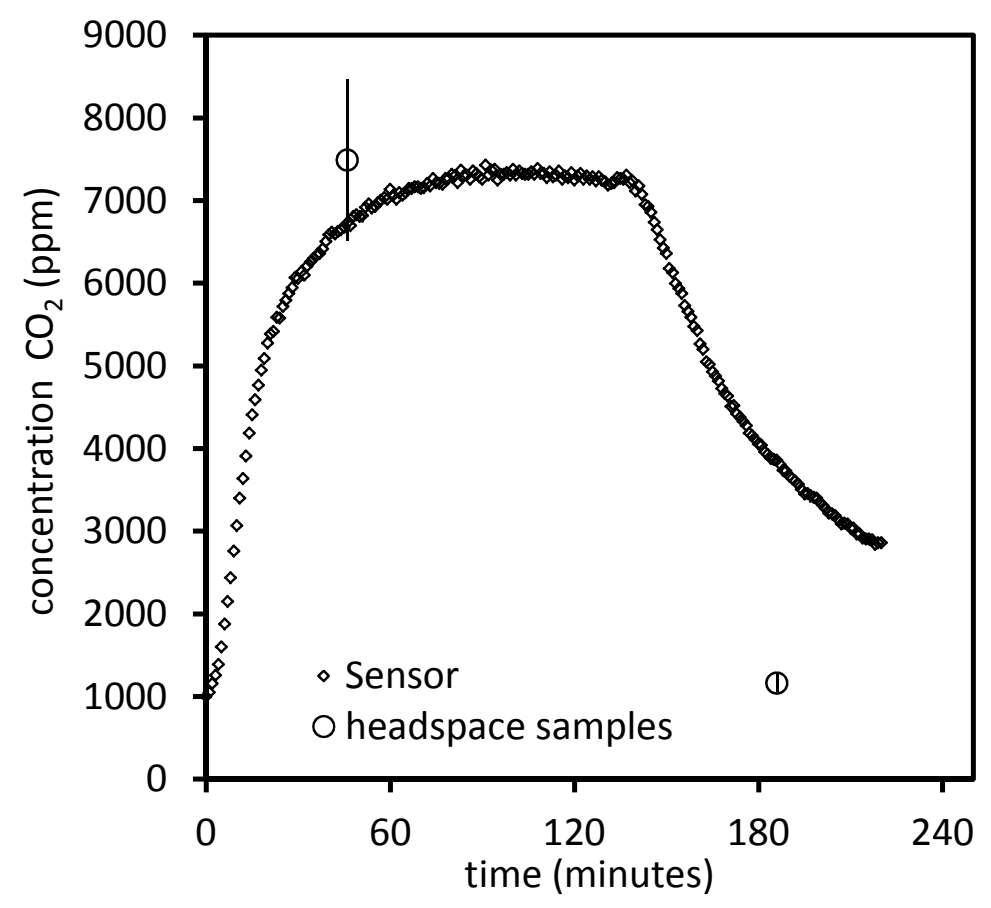

Figure S3: To estimate the response time of silicone-covered $\mathrm{NDI} \mathrm{CO}_{2}$ sensors, about $10 \mathrm{~L}$ of water were filled in a bucket and acidified to $\mathrm{pH} 4$ by adding $1 \mathrm{M} \mathrm{HCl}$. Subsequently $\mathrm{CO}_{2}$ and air were bubbled through the solution from a gas cylinder and using an air pump, respectively, to adjust dissolved $\mathrm{CO}_{2}$ concentrations and mix the water. Headspace samples ( $5 \mathrm{~mL}$ water, $7.5 \mathrm{~mL}$ gas phase) were taken and analyzed by gas chromatography as described in the Methods section. After 124 minutes $\mathrm{CO}_{2}$ supply was stopped but air continuously pumped for another hour. The results suggest that equilibration was $>90 \%$ after one hour with increasing concentration and somewhat more delayed with decreasing concentrations. 


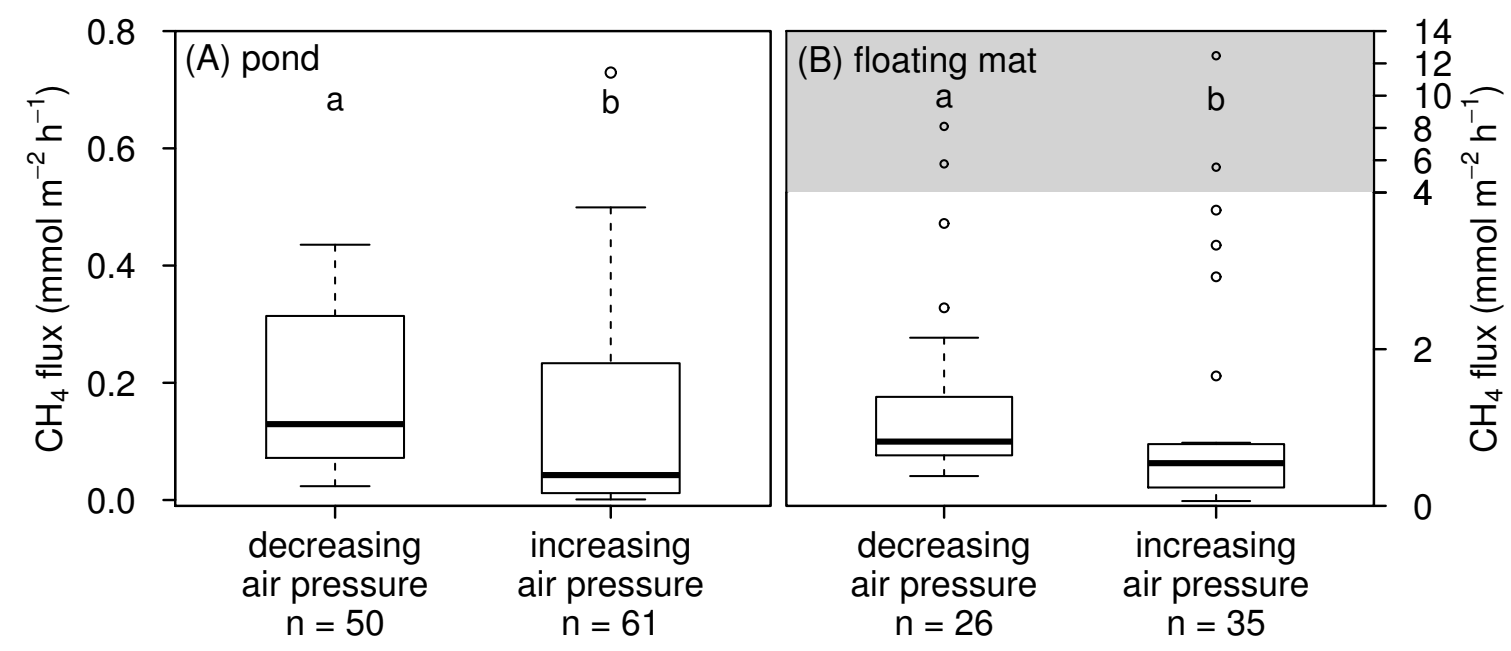

Figure S4: Total $\mathrm{CH}_{4}$ fluxes (i.e. ebullition + diffusive trnasport) during decreasing and an increasing air pressure trends over the last $24 \mathrm{~h}$ for the pond (panel A) and the floating mat (panel B). Different letters indicate significant differences (Kruskal-Wallis test, $p<0.05$ and $p<0.01, n=111$ and $n=61$ ). Note the different scaling of the $y$-axis within the gray area in panel (B). The bold horizontal line shows the median, the bottom and the top of the box the $25^{\text {th }}$ and $75^{\text {th }}$ percentile and the whiskers include all values within 1.5 times the interquartile range. 


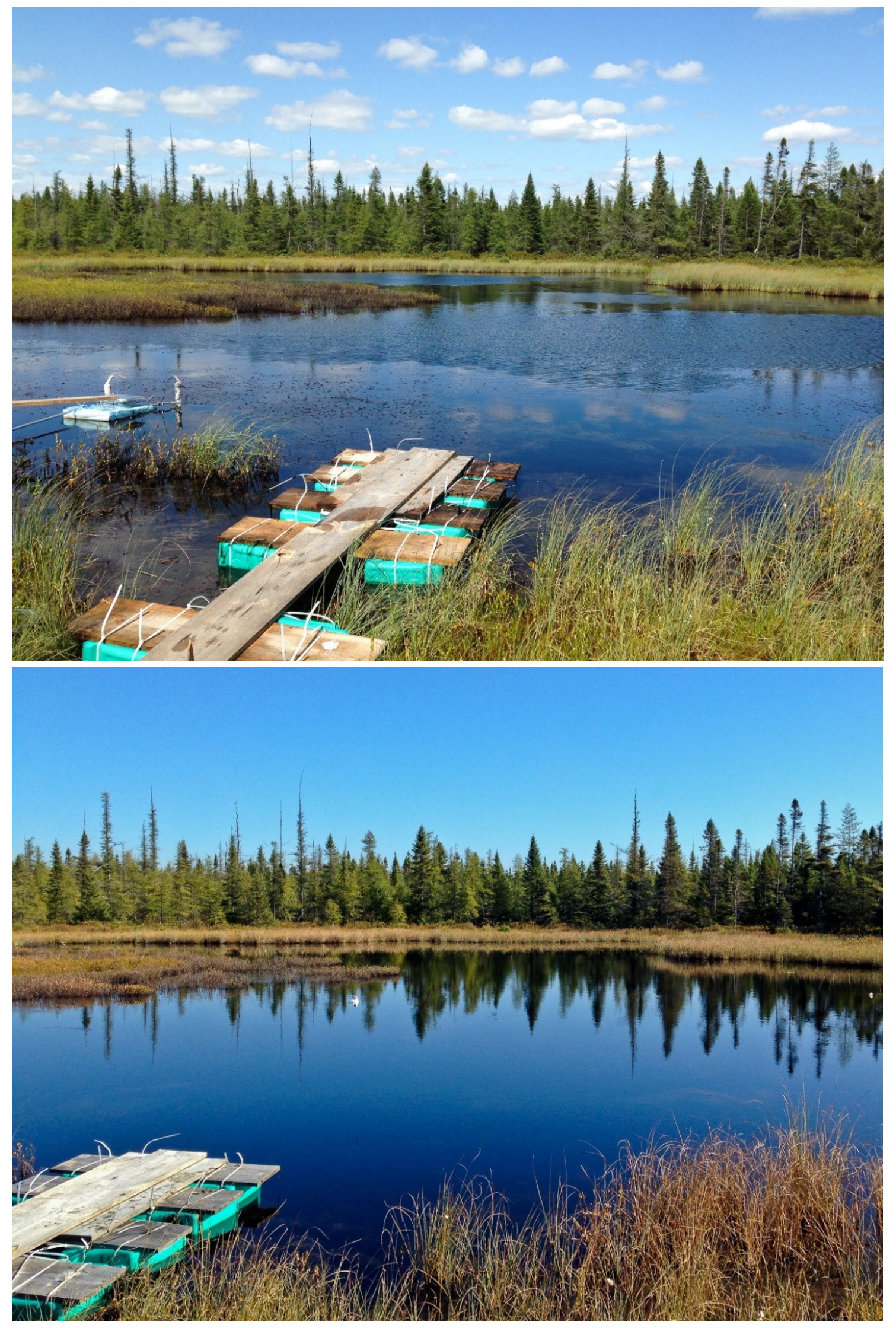

Figure S5: Studied pond with algal mat in the beginning of August 2014 (above) and without algal mat in the end of September 2014 (below). 

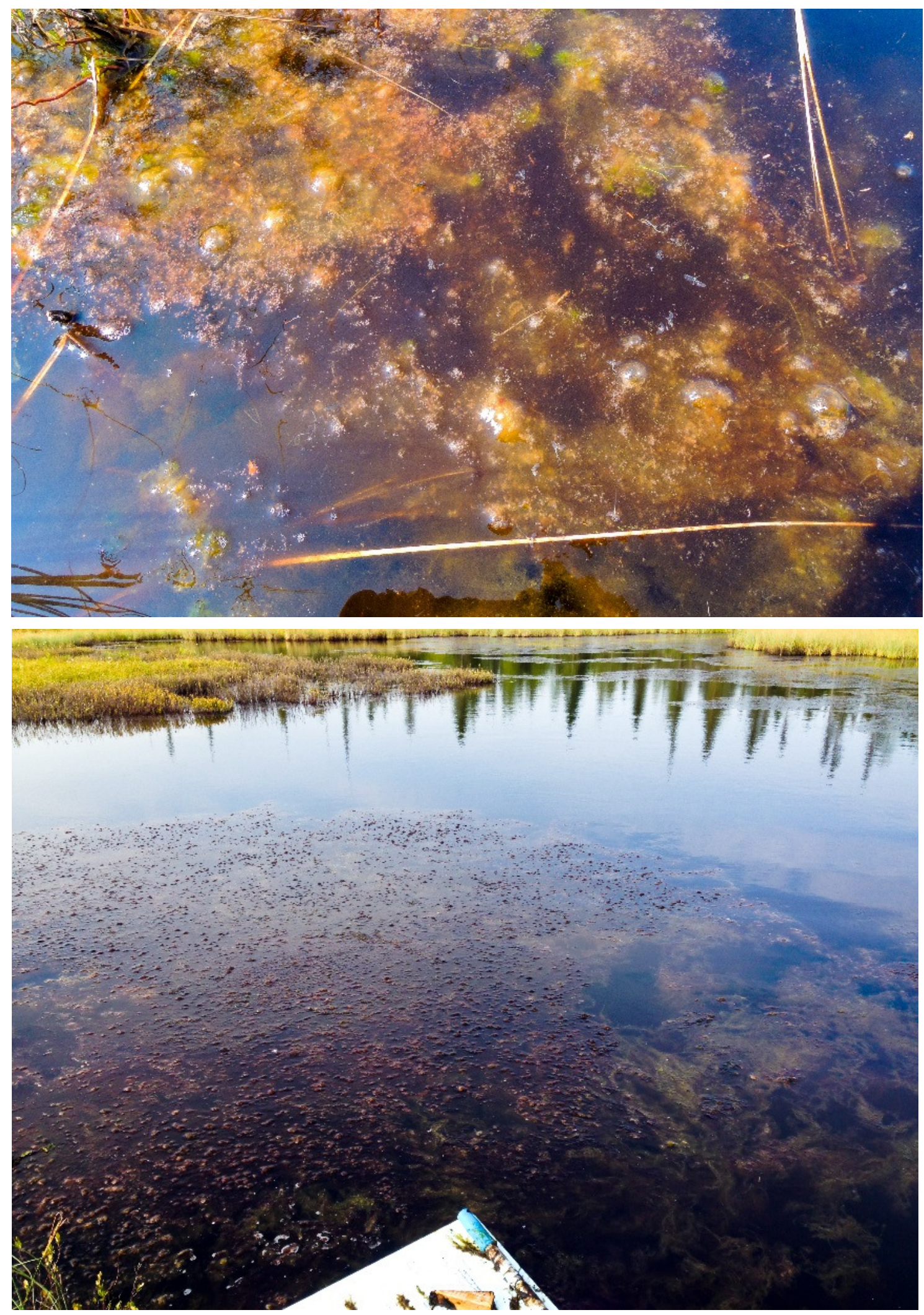

Figure S6: Close-up of algal mat in the beginning of August 2014 (above). 
Table S1. Plant species composition of the floating mat, the surrounding treed area and the chamber measurement plots $\mathbf{m} \mathbf{1}$ to $\mathbf{m} \mathbf{3}$; c: common, s: scattered, r: rare, -: absent; species nomenclature according to Hellquist and Crow (1999a), (1999b), Newmaster and Ragupathy (2012).

\begin{tabular}{|c|c|c|c|c|c|}
\hline \multirow[t]{3}{*}{ Species } & \multicolumn{5}{|c|}{ abundance } \\
\hline & \multirow[b]{2}{*}{ floating mat } & \multirow[b]{2}{*}{ treed area } & \multicolumn{3}{|c|}{ plot } \\
\hline & & & m1 & $\mathrm{m} 2$ & m3 \\
\hline Sphagnum angustifolium (Russ.) C. Jens. & c & $\mathrm{C}$ & c & c & $\mathrm{C}$ \\
\hline Sphagnum magellanicum Brid. & c & $\mathrm{C}$ & - & - & - \\
\hline Sphagnum fuscum (Schimp.) Klinggr. & - & $\mathrm{S}$ & - & - & - \\
\hline Sphagnum wulfianum Girg. & - & $\mathrm{S}$ & - & - & - \\
\hline Larix laricina (DuRoi) K. Koch & - & $c-s$ & - & - & - \\
\hline Picea mariana (Mill.) BSP. & - & $c-s$ & - & - & - \\
\hline Pinus strobus L. & - & $\mathrm{R}$ & - & - & - \\
\hline Eleocharis smallii Britt. & - & $\mathrm{S}$ & - & - & - \\
\hline Eriophorum vaginatum $\mathrm{L}$. & s & $\mathrm{S}$ & - & - & - \\
\hline Eriophorum virginicum $\mathrm{L}$. & s & $\mathrm{S}$ & - & - & - \\
\hline Dulichium arundinaceum (L.) Britt. & $r$ & - & - & - & - \\
\hline Rhynchospora alba (L.) Vahl & c & - & s & c-s & $\mathrm{s}$ \\
\hline Carex aquatilis Wahlenb. & s & - & $r$ & - & $\mathrm{s}$ \\
\hline Carex oligosperma Michx. & s & - & - & - & - \\
\hline Carex magellanica Lam. & $\mathrm{s}$ & - & - & - & - \\
\hline Carex disperma Dew. & - & $\mathrm{S}$ & - & - & - \\
\hline Myrica gale L. & $r$ & $s-r$ & - & - & - \\
\hline Betula pumila $\mathrm{L}$. & - & $S$ & - & - & - \\
\hline Sarracenia purpurea $\mathrm{L}$. & s & - & - & $r$ & - \\
\hline Drosera rotundifolia $\mathrm{L}$. & $r$ & - & - & - & - \\
\hline Populus sp. & - & $\mathrm{S}$ & - & - & - \\
\hline Rhododendron groenlandicum (Oeder) Kron \& Judd & r & $\mathrm{C}$ & - & - & - \\
\hline Kalmia polifolia Wang. & $s$ & $S$ & - & $r$ & - \\
\hline Kalmia angustifolia $\mathrm{L}$. & - & $\mathrm{R}$ & - & - & - \\
\hline Andromeda glaucophylla Link & s & $\mathrm{S}$ & - & $r$ & - \\
\hline Vaccinium oxycoccos $\mathrm{L}$. & $\mathrm{s}$ & $\mathrm{S}$ & - & - & $r$ \\
\hline Vaccinium myrtilloides Hook. & - & $S$ & - & - & - \\
\hline Vaccinium macrocarpon Ait. & - & $\mathrm{S}$ & - & - & - \\
\hline Vaccinium corymbosum L. & - & $\mathrm{S}$ & - & - & - \\
\hline Vaccinium uliginosum L. & - & $\mathrm{R}$ & - & - & - \\
\hline Chamaedaphne calyculata (L.) Moench & $\mathrm{s}$ & $\mathrm{c}-\mathrm{s}$ & $\mathrm{s}$ & $\mathrm{s}$ & $\mathrm{s}$ \\
\hline Aronia melanocarpa (Michx.) Ell. & - & $S$ & - & - & - \\
\hline Cypripedium parviflorum Salisb. & - & $\mathrm{R}$ & - & - & - \\
\hline Cypripedium reginae Walt. & - & $\mathrm{R}$ & - & - & - \\
\hline
\end{tabular}


Table S2. Environmental variables measured at the study site with the corresponding instruments, measuring height, orientation, temporal resolution and accuracy.

\begin{tabular}{|c|c|c|c|c|c|}
\hline Variable & Instrument & $\begin{array}{l}\text { measuring } \\
\text { height or } \\
\text { depth }^{\mathrm{a}}\end{array}$ & $\begin{array}{l}\text { orienta } \\
\text { tion }\end{array}$ & $\begin{array}{l}\text { temporal } \\
\text { resolution }\end{array}$ & accuracy \\
\hline $\begin{array}{l}\text { air temperature } \\
\left({ }^{\circ} \mathrm{C}\right)\end{array}$ & $\begin{array}{l}\text { Temperature/RH Smart } \\
\text { Sensor, S-THB-M002, } \\
\text { Onset }\end{array}$ & $+2.0 \mathrm{~m}$ & north & $5 \mathrm{~min}$ & $\pm 0.21{ }^{\circ} \mathrm{C}$ \\
\hline $\begin{array}{l}\text { relative humidity } \\
(\%)\end{array}$ & $\begin{array}{l}\text { Temperature/RH Smart } \\
\text { Sensor, S-THB-M002, } \\
\text { Onset }\end{array}$ & $+2.0 \mathrm{~m}$ & north & $5 \mathrm{~min}$ & $\pm 2.5 \%$ \\
\hline wind speed $\left(\mathrm{m} \mathrm{s}^{-1}\right)$ & $\begin{array}{l}\text { Wind Speed Smart } \\
\text { Sensor, S-WSA-M002, } \\
\text { Onset }\end{array}$ & $+2.5 \mathrm{~m}$ & $\begin{array}{l}\text { west- } \\
\text { southw } \\
\text { est }\end{array}$ & $5 \mathrm{~min}$ & $\begin{array}{l} \pm 1.1 \mathrm{~m} \mathrm{~s}^{-1} \text { or } \\
4 \% \text { (whichever } \\
\text { is greater) }\end{array}$ \\
\hline wind direction $\left({ }^{\circ}\right)$ & $\begin{array}{l}\text { Wind Direction Smart } \\
\text { Sensor, S-WDA-M002, } \\
\text { Onset }\end{array}$ & $+2.5 \mathrm{~m}$ & $\begin{array}{l}\text { east- } \\
\text { northe } \\
\text { ast }\end{array}$ & $5 \min$ & $\pm 5^{\circ}$ \\
\hline $\begin{array}{l}\text { photosynthetically } \\
\text { active radiation } \\
\left(\mu \mathrm{mol} \mathrm{m}^{-2} \mathrm{~s}^{-1}\right)\end{array}$ & $\begin{array}{l}\text { Photosynthetic Light } \\
\text { (PAR) Smart Sensor, S- } \\
\text { LIA-M002, Onset }\end{array}$ & $+2.3 \mathrm{~m}$ & south & $5 \min$ & $\begin{array}{l} \pm 5 \mu \mathrm{mol} \mathrm{m} \mathrm{m}^{-2} \mathrm{~s}^{-1} \\
\text { or } 5 \% \\
\text { (whichever is } \\
\text { greater) }\end{array}$ \\
\hline precipitation (mm) & $\begin{array}{l}.2 \mathrm{~mm} \text { Rainfall Smart } \\
\text { Sensor, S-RGB-M002, } \\
\text { Onset }\end{array}$ & $+1.0 \mathrm{~m}$ & $\begin{array}{l}\text { west- } \\
\text { northw } \\
\text { est }\end{array}$ & $5 \min$ & $\pm 1 \%$ \\
\hline $\begin{array}{l}\text { water temperature } \\
\text { pond }\left({ }^{\circ} \mathrm{C}\right)\end{array}$ & CTD-Diver, Schlumberger & $-0.2 \mathrm{~m}$ & - & $5 \mathrm{~min}$ & $\pm 0.1^{\circ} \mathrm{C}$ \\
\hline $\begin{array}{l}\text { temperature floa- } \\
\text { ting mat }\left({ }^{\circ} \mathrm{C}\right)\end{array}$ & $\begin{array}{l}\text { Air/Water/Soil Temp } \\
\text { Sensor, TMC6-HD, Onset }\end{array}$ & $\begin{array}{l}-5 \text { and } \\
-10 \mathrm{~cm}\end{array}$ & - & $5 \mathrm{~min}$ & $\pm 0.25^{\circ} \mathrm{C}$ \\
\hline air pressure $(\mathrm{kPa})$ & $\begin{array}{l}\text { Enclosed Path } \mathrm{CO}_{2} / \mathrm{H}_{2} \mathrm{O} \\
\text { Analyzer, LI-7200, LI-COR }\end{array}$ & $+5.0 \mathrm{~m}$ & - & $30 \mathrm{~min}$ & $\pm 4 \%$ \\
\hline
\end{tabular}

${ }^{a}$ : indicated by positive (height) or negative sign (depth)

Table S3. Distance from the floating mat and water depth of the chamber measurement plots of the pond.

\begin{tabular}{lrr}
\hline plot & $\begin{array}{r}\text { distance from the } \\
\text { floating mat }(\mathbf{m})\end{array}$ & water depth (m) \\
\hline p1 & 4.55 & 0.73 \\
p2 & 3.76 & 0.83 \\
p3 & 3.15 & 0.77 \\
p4 & 1.91 & 0.62 \\
p5 & 0.80 & 0.52 \\
p6 & 0.73 & 0.42 \\
\hline
\end{tabular}


Table S4. $\mathrm{CH}_{4}$ fluxes from the studied peatland, floating mat and pond in comparison to $\mathrm{CH}_{4}$ fluxes reported from northern peatlands, floating mats and ponds in literature.

\begin{tabular}{|c|c|c|c|c|c|c|c|}
\hline \multirow[t]{2}{*}{ System } & \multirow[t]{2}{*}{ Location } & \multicolumn{4}{|c|}{$\mathrm{CH}_{4}$ fluxes $\left(\mathrm{mmol} \mathrm{m}^{-2} \mathrm{~h}^{-1}\right)$} & \multirow{2}{*}{$\begin{array}{l}\text { time } \\
\text { horizon }\end{array}$} & \multirow[t]{2}{*}{ reference } \\
\hline & & $\begin{array}{r}\text { mini } \\
\text { mum }\end{array}$ & $\begin{array}{r}\text { me } \\
\text { dian }\end{array}$ & mean & $\begin{array}{l}\text { maxi } \\
\text { mum }\end{array}$ & & \\
\hline $\begin{array}{l}\text { Peatlands } \\
\text { peatland site, Wylde } \\
\text { Lake Bog }\end{array}$ & Ontario, Canada & 0.00 & 0.32 & 0.71 & 28.13 & $\begin{array}{l}\text { July to } \\
\text { Sept. }\end{array}$ & this study \\
\hline Bog & Ontario, Canada & & & 0.07 & & $\begin{array}{l}\text { July and } \\
\text { Aug. }\end{array}$ & $\begin{array}{l}\text { Dinsmore et } \\
\text { al. (2009) }\end{array}$ \\
\hline Fen & Michigan, USA & & & 0.02 & & $\begin{array}{l}\text { April to } \\
\text { Oct. }\end{array}$ & $\begin{array}{l}\text { Ballantyne et } \\
\text { al. (2014) }\end{array}$ \\
\hline $\begin{array}{l}\text { fen hummock } \\
\text { fen lawns and hollows }\end{array}$ & Quebec, Canada & & & $\begin{array}{l}0.07 \\
0.09\end{array}$ & & two years & $\begin{array}{l}\text { Trudeau et } \\
\text { al. (2013) }\end{array}$ \\
\hline 2 fens, 1 bog & Ontario, Canada & & & 0.04 & & $\begin{array}{l}\text { June to } \\
\text { Oct. }\end{array}$ & $\begin{array}{l}\text { Hamilton et } \\
\text { al. (1994) }\end{array}$ \\
\hline poor fen & Quebec, Canada & 0.00 & 0.11 & & 0.38 & $\begin{array}{l}\text { May to } \\
\text { Sept. }\end{array}$ & $\begin{array}{l}\text { Strack et al. } \\
(2006)\end{array}$ \\
\hline $\begin{array}{l}\text { bog hummocks } \\
\text { bog lawns and ponds }\end{array}$ & Ontario, Canada & & & $\begin{array}{l}0.01 \\
0.26\end{array}$ & & May to Oct. & $\begin{array}{l}\text { Moore et al. } \\
(2011)\end{array}$ \\
\hline $\begin{array}{l}\text { floating mats } \\
\text { floating mat in Wylde } \\
\text { Lake Bog }\end{array}$ & Ontario, Canada & 0.06 & 0.64 & 1.52 & 14.98 & $\begin{array}{l}\text { July to } \\
\text { Sept. }\end{array}$ & this study \\
\hline $\begin{array}{l}\text { floating mat on } \\
\text { thermokarst pond in } \\
\text { bog }\end{array}$ & Siberia, Russia & & & 0.14 & & one year & $\begin{array}{l}\text { Flessa et al. } \\
(2008)\end{array}$ \\
\hline $\begin{array}{l}\text { floating mats in bog } \\
\text { Sphagnum } \\
\text { Phragmites australis } \\
\text { Menyanthes trifoliata }\end{array}$ & central Japan & & & $\begin{array}{l}0.18 \\
0.76 \\
1.17\end{array}$ & & $\begin{array}{l}\text { April to } \\
\text { Oct. }\end{array}$ & $\begin{array}{l}\text { Sugimoto } \\
\text { and Fujita } \\
(1997)\end{array}$ \\
\hline $\begin{array}{l}\text { Ponds } \\
\text { pond in Wylde Lake Bog } \\
\text { area: } 847 \mathrm{~m}^{2} \\
\text { depth: } 0.5 \mathrm{~m}\end{array}$ & Ontario, Canada & 0.00 & 0.14 & 0.22 & 2.00 & $\begin{array}{l}\text { July to } \\
\text { Sept. }\end{array}$ & this study \\
\hline $\begin{array}{l}\text { Beaver pond } \\
\text { depth: } 0.8 \mathrm{~m}\end{array}$ & Ontario, Canada & & & 0.01 & & $\begin{array}{l}\text { July and } \\
\text { Aug. }\end{array}$ & $\begin{array}{l}\text { Dinsmore et } \\
\text { al. (2009) }\end{array}$ \\
\hline $\begin{array}{l}2 \text { fen pools } \\
\text { area: } 65 \text { and } 200 \mathrm{~m}^{2} \\
\text { depth: } 0.4 \text { and } 0.9 \mathrm{~m}\end{array}$ & Quebec, Canada & & & $\begin{array}{l}0.50 \\
\text { and } \\
0.23\end{array}$ & & 2 years & $\begin{array}{l}\text { Trudeau et } \\
\text { al. (2013) }\end{array}$ \\
\hline $\begin{array}{l}24 \text { bog and fen ponds } \\
\text { area: } 32 \text { to } 41620 \mathrm{~m}^{2} \\
\text { depth: } 0.1 \text { to } 2.0 \mathrm{~m}\end{array}$ & Ontario, Canada & & & 0.38 & & $\begin{array}{l}\text { June to } \\
\text { Oct. }\end{array}$ & $\begin{array}{l}\text { Hamilton et } \\
\text { al. (1994) }\end{array}$ \\
\hline $\begin{array}{l}\text { fen pond } \\
\text { area: } 10000 \mathrm{~m}^{2} \\
\text { max. depth: } 3.2 \mathrm{~m}\end{array}$ & northern Finland & & & 0.48 & & $\begin{array}{l}\text { May to } \\
\text { Sept. }\end{array}$ & $\begin{array}{l}\text { Huttunen et } \\
\text { al. (2002) }\end{array}$ \\
\hline $\begin{array}{l}5 \text { bog pools } \\
\text { area: } 128 \text { to } 2563 \mathrm{~m}^{2} \\
\text { depth: } 0.4 \text { to } 2.0 \mathrm{~m}\end{array}$ & Quebec, Canada & $\begin{array}{r}0.00 \\
\text { to } \\
0.01\end{array}$ & & $\begin{array}{r}0.01 \\
\text { to } \\
0.20\end{array}$ & $\begin{array}{r}0.04 \\
\text { to } \\
0.41\end{array}$ & 1.5 years & $\begin{array}{l}\text { Pelletier et } \\
\text { al. (2014) }\end{array}$ \\
\hline $\begin{array}{l}\text { fen pond } \\
\text { area: } 5000 \mathrm{~m}^{2} \\
\text { depth: } 1 \mathrm{~m}\end{array}$ & Siberia, Russia & 0.03 & 0.06 & 0.11 & 0.31 & $\begin{array}{l}\text { July to } \\
\text { Sept. }\end{array}$ & $\begin{array}{l}\text { Repo et al. } \\
(2007)\end{array}$ \\
\hline
\end{tabular}


Table S5. Daytime maximum net ecosystem exchange (NEE) and ecosystem respiration (ER) of the studied peatland and floating mat in comparison to respective values from temperate peatlands reported in literature.

\begin{tabular}{|c|c|c|c|c|c|c|c|}
\hline \multirow[t]{2}{*}{ System } & \multirow[t]{2}{*}{ Location } & \multicolumn{4}{|c|}{ maximum NEE and ER $\left(\mathrm{mmol} \mathrm{m}^{-2} \mathrm{~h}^{-1}\right)$} & \multirow{2}{*}{$\begin{array}{l}\text { time } \\
\text { horizon }\end{array}$} & \multirow[t]{2}{*}{ reference } \\
\hline & & $\begin{array}{r}\operatorname{minimu} \\
\mathrm{m}\end{array}$ & median & Mean & $\begin{array}{l}\text { maxi } \\
\text { mum }\end{array}$ & & \\
\hline peatland site, & Ontario, Canada & -36.96 & -16.98 & -18.73 & -8.10 & July to & this study \\
\hline Wylde Lake Bog & & 2.61 & 11.98 & 13.59 & 36.93 & Sept. & \\
\hline floating mat in & Ontario, & -11.46 & -4.81 & -4.40 & 0.71 & July to & this study \\
\hline Wylde Lake Bog & Canada & 0.53 & 6.77 & 6.41 & 13.45 & Sept. & \\
\hline \multirow[t]{2}{*}{ Bog } & Ontario, Canada & & & -29.7 & & May to & Larmola et \\
\hline & & & & 15.3 & & Sept. & al. (2013) \\
\hline \multirow[t]{2}{*}{ Fen } & Michigan, USA & & & -7.56 & & April to & Ballantyne \\
\hline & & & & 8.64 & & Oct. & et al. (2014) \\
\hline
\end{tabular}

Table S6. $\mathrm{CO}_{2}$ fluxes from the studied pond in comparison to $\mathrm{CO}_{2}$ fluxes from ponds in northern peatlands reported in literature.

\begin{tabular}{|c|c|c|c|c|c|c|c|}
\hline \multirow[t]{2}{*}{ System } & \multirow[t]{2}{*}{ Location } & \multicolumn{4}{|c|}{$\mathrm{CO}_{2}$ fluxes $\left(\mathrm{mmol} \mathrm{m}^{-2} \mathrm{~h}^{-1}\right)$} & \multirow{2}{*}{$\begin{array}{l}\text { time } \\
\text { horizon }\end{array}$} & \multirow[t]{2}{*}{ reference } \\
\hline & & $\begin{array}{l}\text { mini } \\
\text { mum }\end{array}$ & $\begin{array}{r}\text { medi } \\
\text { an }\end{array}$ & mean & $\begin{array}{l}\text { maxi } \\
\text { mum }\end{array}$ & & \\
\hline $\begin{array}{l}\text { pond in Wylde Lake Bog } \\
\text { area: } 847 \mathrm{~m}^{2} \\
\text { depth: } 0.5 \mathrm{~m}\end{array}$ & $\begin{array}{l}\text { Ontario, } \\
\text { Canada }\end{array}$ & -0.75 & 1.16 & 1.32 & 4.59 & $\begin{array}{l}\text { July to } \\
\text { Sept. }\end{array}$ & this study \\
\hline $\begin{array}{l}\text { Beaver pond } \\
\text { depth: } 0.8 \mathrm{~m}\end{array}$ & $\begin{array}{l}\text { Ontario, } \\
\text { Canada }\end{array}$ & & & 13.48 & & $\begin{array}{l}\text { July and } \\
\text { Aug. }\end{array}$ & $\begin{array}{l}\text { Dinsmore et } \\
\text { al. (2009) }\end{array}$ \\
\hline $\begin{array}{l}24 \text { bog and fen ponds } \\
\text { area: } 32 \text { to } 41620 \mathrm{~m}^{2} \\
\text { depth: } 0.1 \text { to } 2.0 \mathrm{~m}\end{array}$ & $\begin{array}{l}\text { Ontario, } \\
\text { Canada }\end{array}$ & & & 6.96 & & $\begin{array}{l}\text { June to } \\
\text { Oct. }\end{array}$ & $\begin{array}{l}\text { Hamilton et } \\
\text { al. (1994) }\end{array}$ \\
\hline $\begin{array}{l}\text { peatland pond } \\
\text { area: } 10000 \mathrm{~m}^{2} \\
\text { max. depth: } 3.2 \mathrm{~m}\end{array}$ & $\begin{array}{l}\text { northern } \\
\text { Finland }\end{array}$ & & & 0.50 & & $\begin{array}{l}\text { May to } \\
\text { Sept. }\end{array}$ & $\begin{array}{l}\text { Huttunen et } \\
\text { al. (2002) }\end{array}$ \\
\hline $\begin{array}{l}5 \text { bog pools } \\
\text { area: } 128 \text { to } 2563 \mathrm{~m}^{2} \\
\text { depth: } 0.4 \text { to } 2.0 \mathrm{~m}\end{array}$ & $\begin{array}{l}\text { Quebec, } \\
\text { Canada }\end{array}$ & $\begin{array}{r}0.04 \\
\text { to } \\
0.34\end{array}$ & & $\begin{array}{r}0.32 \\
\text { to } \\
0.80\end{array}$ & $\begin{array}{r}0.53 \\
\text { to } \\
1.98\end{array}$ & 1.5 years & $\begin{array}{l}\text { Pelletier et } \\
\text { al. (2014) }\end{array}$ \\
\hline $\begin{array}{l}\text { fen pond } \\
\text { area: } 5000 \mathrm{~m}^{2} \\
\text { depth: } 1 \mathrm{~m}\end{array}$ & $\begin{array}{l}\text { Siberia, } \\
\text { Russia }\end{array}$ & 0.85 & 1.42 & 1.52 & 2.94 & $\begin{array}{l}\text { July to } \\
\text { Sept. }\end{array}$ & $\begin{array}{l}\text { Repo et al. } \\
\text { (2007) }\end{array}$ \\
\hline
\end{tabular}




\section{References}

Ballantyne, D.M., Hribljan, J.A., Pypker, T.G., Chimner, R.A.: Long-term water table manipulations alter peatland gaseous carbon fluxes in Northern Michigan. Wetlands Ecology and Management 22, 35-47, 2014.

Cole, J.J., Caraco, N.F.: Atmospheric exchange of carbon dioxide in a low-wind oligotrophic lake measured by the addition of $\mathrm{SF}_{6}$. Limnology and Oceanography 43, 647-656, 1998.

Crusius, J., Wanninkhof, R.: Gas transfer velocities measured at low wind speed over a lake. Limnology and Oceanography 48, 1010-1017, 2003.

Dinsmore, K.J., Billett, M.F., Moore, T.R.: Transfer of carbon dioxide and methane through the soil-water-atmosphere system at Mer Bleue peatland, Canada. Hydrological Processes 23, 330-341, 2009.

Flessa, H., Rodionov, A., Guggenberger, G., Fuchs, H., Magdon, P., Shibistova, O., Zrazhevskaya, G., Mikheyeva, N., Kasansky, O., Blodau, C.: Landscape controls of $\mathrm{CH}_{4}$ fluxes in a catchment of the forest tundra ecotone in northern Siberia. Global Change Biology 14, 2040-2056, 2008.

Hamilton, J.D., Kelly, C.A., Rudd, J.W.M., Hesslein, R.H., Roulet, N.T.: Flux to the atmosphere of $\mathrm{CH}_{4}$ and $\mathrm{CO}_{2}$ from wetland ponds on the Hudson Bay lowlands (HBLs). Journal of Geophysical Research 99, 1495-1510, 1994.

Hellquist, C.B., Crow, G.E.: Aquatic and Wetland Plants of Northeastern North America, A Revised and Enlarged Edition of Norman C. Fassett's A Manual of Aquatic Plants, Volume II Angiosperms Monocotyledons. Madison, 1999a.

Hellquist, C.B., Crow, G.E.: Aquatic and Wetland Plants of Northeastern North America, A Revised and Enlarged Edition of Norman C. Fassett's A Manual of Aquatic Plants, Volume I Pteridophytes, Gymnosperms, and Angiosperms Dicotyledons. Madison, 1999b.

Huttunen, J.T., Väisänen, T.S., Heikkinen, M., Hellsten, S., Nykänen, H., Nenonen, O., Martikainen, P.J.: Exchange of $\mathrm{CO}_{2}, \mathrm{CH}_{4}$ and $\mathrm{N}_{2} \mathrm{O}$ between the atmosphere and two northern boreal ponds with catchments dominated by peatlands or forests. Plant and Soil 242, 137146, 2002. 
Larmola, T., Bubier, J.L., Kobyljanec, C., Basiliko, N., Juutinen, S., Humphreys, E.R., Preston, M., Moore, T.R.: Vegetation feedbacks of nutrient addition lead to a weaker carbon sink in an ombrotrophic bog. Global Change Biology 19, 3729-3739, 2013.

Moore, T.R., De Young, A., Bubier, J.L., Humphreys, E.R., Lafleur, P.M., Roulet, N.T.: A MultiYear Record of Methane Flux at the Mer Bleue Bog, Southern Canada. Ecosystems 14, 646657, 2011.

Newmaster, S.G., Ragupathy, S.: Flora Ontario - Integrated Botanical Information System (FOIBIS). University of Guelph. URL http://www.uoguelph.ca/foibis/ (accessed April $24^{\text {th }}$, 2015), 2012.

Pelletier, L., Strachan, I.B., Garneau, M., Roulet, N.T.: Carbon release from boreal peatland open water pools: Implication for the contemporary C exchange. Journal of Geophysical Research: Biogeosciences 119, 207-222, 2014.

Repo, M.E., Huttunen, J.T., Naumov, A. V., Chichulin, A. V., Lapshina, E.D., Bleuten, W., Martikainen, P.J.: Release of $\mathrm{CO}_{2}$ and $\mathrm{CH}_{4}$ from small wetland lakes in western Siberia. Tellus 59B, 788-796, 2007.

Sander, R.: Compilation of Henry's Law Constants for Inorganic and Organic Species of Potential Importance in Environmental Chemistry. Max-Planck Institute of Chemistry, Mainz, 1999.

Singh, S., Bhatti, T.S., Kothari, D.P.: Wind Power Estimation Using Artificial Neural Network. Journal of Energy Engineering 133, 46-52, 2007.

Strack, M., Waller, M.F., Waddington, J.M.: Sedge succession and peatland methane dynamics: A potential feedback to climate change. Ecosystems 9, 278-287, 2006.

Sugimoto, A., Fujita, N.: Characteristics of methane emissions from different vegetations on a wetland. Tellus 49B, 382-392, 1997.

Trudeau, N.C., Garneau, M., Pelletier, L.: Methane fluxes from a patterned fen of the northeastern part of the La Grande river watershed, James Bay, Canada. Biogeochemistry 113, 409-422, 2013.

Wanninkhof, R.: Relationship Between Wind Speed and Gas Exchange Over the Ocean. Journal of Geophysical Research 97, 7373-7382, 1992. 